\title{
Knowledge, Attitude, and Practice of General Population Towards CVD in Saudi Arabia
}

\author{
Authors \\ Mohammad Sami Basubrain ${ }^{1}$, Esraa Sami Basubrain ${ }^{1}$, Lama Rashid Elessawi ${ }^{1}$, \\ Omar Ahmed Bafarat ${ }^{1}$, Rana Abdulrahman Baflah ${ }^{1}$, Rania Mohammed Srouji ${ }^{1}$, \\ Omar Sami Basubrain ${ }^{2}$, Hassnah Omar Dammas ${ }^{2}$ \\ ${ }^{1}$ Ministry of Health, Jeddah, KSA \\ ${ }^{2}$ King Abdullah Medical City (KAMC-HC), Makkah, KSA \\ Corresponding Author \\ Dr Mohammad Sami Basubrain \\ Email: mohammed@basubrain.com
}

\begin{abstract}
According to American Board of Preventive Medicine: "Preventive Medicine is the specialty of medical practice that focuses on the health of individuals, communities, and defined populations. Its goal is to protect, promote, and maintain health and well-being and to prevent disease, disability, and death" [1].

Objective: To highlight the relationship between CVD risk factors knowledge and the attitude of the general population towards the preventive practices.

Methods: This survey was done using a third party online survey agent, it was targeting the general population of KSA.

Conclusion: By the end of this study we found that the general healthy population has inadequate knowledge about the risk factors and their relationship with CVD. Despite that our patients have good knowledge about the relationship between their disease and the risk for CVD, except DM patient, they didn't translate it to right attitude and behaviors.
\end{abstract}

\section{INTRODUCTION}

According to the American Board of Preventive Medicine: "Preventive Medicine is the specialty of medical practice that focuses on the health of individuals, communities, and defined populations. Its goal is to protect, promote, and maintain health and well-being and to prevent disease, disability, and death" ${ }^{[1]}$.

Cardiovascular diseases (CVDs) "are a group of disorders that involve the heart or blood vessels or both. They include coronary heart disease (CHD), cerebrovascular disease, peripheral arterial disease, rheumatic heart disease, congenital heart disease, and deep vein thrombosis and pulmonary embolism" ${ }^{[2]}$.

CVDs are the number one cause of death globally: more people die annually from CVDs than from any other cause. It is estimated that 17.5 million people died from CVDs in 2012, representing $31 \%$ of all global deaths. Of these deaths, an estimated 7.4 million were due to coronary heart disease and 6.7 million were due to stroke ${ }^{[3]}$.

By 2030, almost 23.6 million people will die from CVDs, mainly from heart disease and stroke. These 
are projected to remain the single leading causes of death $^{[4]}$.

The risk of developing CVD depends to a large extent on the presence of several risk factors. The major risk factors for CVD include tobacco use, high blood pressure, high blood glucose, lipid abnormalities, obesity, and physical inactivity. The global variations in CVD rates are related to temporal and regional differences in these known risk factors ${ }^{[5]}$.

A notable decline in cardiovascular deaths has been shown as a result of controlling modifiable risk factors ${ }^{[6]}$, which apparently emphasize the role of preventive medicine.

\section{METHODS}

This survey was targeting the general population, Saudi and non-Saudi, who are living in Kingdom of Saudi Arabia. All participants are over 20 years old. It is targeting both male and female regardless of working in the medical field or not. Data was collected through third party online survey agent, with a restricted IP to prevent double entries, using a cross-sectional method.

\section{RSULTS}

Data was collected from 1,178 participants living in KSA. $81.32 \%$ of them are female. $89.47 \%$ of them are not working in the medical field. All the subjects are over 20 years old. $59.59 \%$ have a bachelor degree, $17.06 \%$ have a postgraduate university degree and $16.81 \%$ with high school degree.

In this population, there were $32.09 \%$ with chronic diseases, $40.37 \%$ with HTN, $35.88 \%$ with DM, $33.51 \%$ with high cholesterol and $10.03 \%$ how already developed CVD.

In this survey $79.97 \%$ of the participants are nonsmokers, $14.35 \%$ are current smokers, and $5.69 \%$ are Ex-Smokers.

Risk factors Knowledge and Preventive behavior This survey showed that $46.86 \%$ of total population are aware of preventive medicine and its practices in general, $52.62 \%$ knew about it through social media and $15.01 \%$ from a physician. $99.04 \%$ of the total population support that we should have like these programs. $52.80 \%$ of the total population knows that all the governmental primary health care centers have a chronic disease clinic.

\section{General Knowledge of Risk Factors General Population}

$77.33 \%$ of the general population knew that obesity is a risk factor for CVD, which represent the highest percent. Surprisingly only $36.93 \%$ knew that DM is a risk factor. Table1 illustrates the rest of the risk factors.

\section{Healthy Population}

Again, $76.88 \%$ of them recognized obesity as a risk factor for CVD, yet only $33 \%$ knew that DM is also a risk factor. Table1illustrates the rest of the risk factors.

Table 1 General Knowledge of CVD Risk Factors

\begin{tabular}{|l|c|c|}
\hline & General Population & Healthy Population \\
\hline Family History & $35.31 \%$ & $33.88 \%$ \\
\hline Dyslipidaemia & $70.97 \%$ & $69.75 \%$ \\
\hline Diabetes & $36.93 \%$ & $33 \%$ \\
\hline Hypertension & $61.38 \%$ & $58 \%$ \\
\hline Smoking & $63.92 \%$ & $63.25 \%$ \\
\hline Bad Food Habits & $61.12 \%$ & $61.12 \%$ \\
\hline Inactivity & $66.21 \%$ & $67.13 \%$ \\
\hline Obesity & $77.33 \%$ & $76.88 \%$ \\
\hline Do not Know & $4.92 \%$ & $4.63 \%$ \\
\hline
\end{tabular}

\section{Chronic Disease Patients}

Regarding chronic disease participants who recognize that their disease is a CVD risk factors, the highest number of awareness was noticed among HTN patients who represent $82.35 \%$ of them, followed by $81.10 \%$ in dyslipidemia patients and $65.44 \%$ of DM Patients.

\section{Knowledge and attitude toward individual risk factors \\ Smoking}

Current smokers' participants reported that $85.88 \%$ have had received a helpful information about smoking risks. $70.34 \%$ said it was useful, but it did not help them to quit Table2.

For those who are a former smoker's, $55.22 \%$ of them received information to stop smoking, and 
$94.59 \%$ mentioned that it helped them in quitting smoking. Table 2

Table 2 Smoking Cessation Awareness

\begin{tabular}{|l|c|c|c|}
\hline & Smoker & Ex-Smoker & Non Smoker \\
\hline In population & $14.35 \%$ & $5.69 \%$ & $79.97 \%$ \\
\hline Get helpful info & $85.88 \%$ & $55.22 \%$ & $35.14 \%$ \\
\hline Was useful & $70.34 \%$ & $94.59 \%$ & \\
\hline
\end{tabular}

In $57.24 \%$ of current smokers, such advice was received from awareness campaigns and only $31.03 \%$ from a physician. Similarly, only $37.84 \%$ of ex-smokers got awareness from a doctor, $32.43 \%$ from awareness campaigns, and $35.14 \%$ from friends. Table 3

Table 3 Smoking Cessation Advice Sources

\begin{tabular}{|l|c|c|}
\hline & Smoker & Ex-Smoker \\
\hline Physician & $31.03 \%$ & $37.84 \%$ \\
\hline TV shows & $51.03 \%$ & $27.03 \%$ \\
\hline Cigarettes Pack & $46.21 \%$ & $24.32 \%$ \\
\hline Internet & $46.90 \%$ & $29.73 \%$ \\
\hline Campaign & $57.24 \%$ & $32.43 \%$ \\
\hline Friends & $46.21 \%$ & $35.14 \%$ \\
\hline
\end{tabular}

Given the number of current and former smokers, we looked into the degree of awareness and application of screening recommendations for Abdominal Aortic Aneurysm, despite the fact that it is not considered as a CVD. Only $33.33 \%$ of smokers and former smokers over the age 65 years had abdominal ultrasonography based on physician's order for other complaint andnot as a screening method for the detection Abdominal Aortic Aneurysm. In fact, 50\% ofthose who did not do it claimed that it is not beneficial. Surprisingly, only $2.38 \%$ of total population in thissurvey had a previous education about abdominal aortic aneurysm.

\section{HTN}

The majority of hypertension free population, $72.66 \%$, don't measure their blood pressure regularly. The main reasons reported are the lack of knowledge in $48.49 \%$ and lack of purpose in $33.05 \%$. 85.10\% knowsthe ideal blood pressure.

For hypertensive patients, $90.85 \%$ knew the ideal blood pressure and $82.35 \%$ knew that HTN is one of the risk factors of CVD yet only $60.78 \%$ are measuring their blood pressure routinely. In $33.33 \%$ of the remaining hypertensive patients attributes lack of follow up to lack of motivation and in $30 \%$ to lack of knowledge of the need for regular checks.

\section{DM}

$89.84 \%$ of all non-diabetic subjects who were over 40 years old had their blood glucose level tested. In $52.56 \%$ of them it was upon a physician's order and in $29.45 \%$ did it as routine. Of note, $85.71 \%$ of those who did not do the blood test Presumed they did not have to test.

As for the diabetic participants in this survey, $25.74 \%$ have a controlled blood sugar while the remaining $41.91 \%, 32.35 \%$ Consecutively are not controlled and sometimes controlled. The reason mainly was due to an unhealthy lifestyle in the majority of the subjects, poor diet control, and inactivity scored $71.29 \%$ and $61.39 \%$ respectively. Furthermore, $22.77 \%$ are not following it with a physician. As has been mentioned previously $65.44 \%$ of the diabetic patients aware that DM could cause CVD in the future.

\section{Dyslipidaemia}

$66.74 \%$ of non-dyslipidaemia participants had their cholesterol level tested as recommended by the USPSTF. $32.38 \%$ of them did itupon a physician's order, while $36.65 \%$ did it incidentally. The remaining $33.26 \%$ did not do it mainly because they don't realize that they must do it in $65.71 \%$ of them. On the other hand, only $70.56 \%$ of non-dyslipidaemia participants have a previous knowledge that dyslipidemia is a risk factor for CVD.

Regarding our dyslipidemic participants, 80.10\% knew that dyslipidemia is a risk factor for CVD and $79.53 \%$ are following up in a clinic, which represent the highest percent of knowledge and follow-up among the survey participants.

\section{Exercise}


Exercise is considered one of the most important interventions to reduce CVD risk. Only $11.71 \%$ of total population exercise regularly, $52.63 \%$ occasionally and $35.65 \%$ do not exercise at all. For those who exercise regularly, $10.14 \%$ do it less than three days a week and $9.42 \%$ do it less than $30 \mathrm{~min}$ per exercise.

As to those who don't or occasionally exercise, $60.10 \%$ are not interested in exercising, and $44.42 \%$ don't have time.

CVD risk factors are multiple, and the accumulation of more than one risk factor impose a higher risk for that case to develop CVD in the future. So we looked into the percent of subjects with one risk factor in addition to the lack of exercise, we found that only $11.03 \%, 13.07 \%, 5.51 \%$ of DM, HTN, and high cholesterol level subject, consecutively, exercise regularly. Table 4

Table 4 (exercise among participants)

\begin{tabular}{|l|c|c|c|}
\hline & Regular exercise & No exercise & $\begin{array}{c}\text { Occasional } \\
\text { exercise }\end{array}$ \\
\hline Population & $11.71 \%$ & $35.65 \%$ & $52.63 \%$ \\
\hline CVD & $10.53 \%$ & $42.11 \%$ & $47.37 \%$ \\
\hline DM & $11.03 \%$ & $47.79 \%$ & $41.18 \%$ \\
\hline HTN & $13.07 \%$ & $40.52 \%$ & $46.41 \%$ \\
\hline CHLO. & $5.51 \%$ & $48.03 \%$ & $46.46 \%$ \\
\hline
\end{tabular}

\section{Follow up Behavior}

The healthy participants of this survey reported that they visit doctors or clinics when they are sick only in $87.75 \%$. Only $16.13 \%$ of them had a routine check-up.

Subjects with risk factors showed good follow up with $73.53 \%, 76.32 \%, 78.43 \%$, and $79.53 \%$ of DM, CVD patients, HTN, and dyslipidemia consecutively had a regular check-up.

Lack of interest was the most common reason for not following up among all participants, the highest $69.23 \%$ being among patients with dyslipidemia. Table5

Table 5 Reasons for Lack of Routine Follow-up

\begin{tabular}{|l|c|c|c|c|}
\hline & CVD & Cholesterol & HTN & DM \\
\hline Not Required & $22.2 \%$ & $23.08 \%$ & $21.21 \%$ & $13.89 \%$ \\
\hline $\begin{array}{l}\text { Dislike Doctor } \\
\text { Advice }\end{array}$ & 0 & $7.69 \%$ & $3.03 \%$ & $11.11 \%$ \\
\hline
\end{tabular}

\begin{tabular}{|l|c|c|c|c|}
\hline No Time & $22.22 \%$ & $11.54 \%$ & $15.15 \%$ & $16.67 \%$ \\
\hline Transportation & $11.11 \%$ & $7.69 \%$ & 0 & $2.78 \%$ \\
\hline Cost & $33.3 \%$ & $7.69 \%$ & $9.09 \%$ & $13.89 \%$ \\
\hline Lack of Interest & $44.4 \%$ & $69.2 \%$ & $51.52 \%$ & $55.56 \%$ \\
\hline Other & $11.1 \%$ & $7.69 \%$ & $12.1 \%$ & $16.67 \%$ \\
\hline
\end{tabular}

\section{DISCUSSION}

CVDs are the number one cause of death globally with more people dying annually from CVDs than from any other cause. It is estimated that 17.5 million people died from CVDs in 2012, which represents $31 \%$ of all global deaths. Of these deaths, 7.4 million were due to coronary heart disease and 6.7 million were due to stroke ${ }^{[3]}$.

According to WHO "Cardiovascular diseases (CVDs) are a group of disorders of the heart and blood vessels, and they include: coronary heart disease, cerebrovascular disease, peripheral arterial disease, rheumatic heart disease, congenital heart disease, deep vein thrombosis and pulmonary embolism",[1].

DM, HTN, dyslipidemia, Smoking and lack of exercise are modifiable risk factors for CVD. In this survey, there were more than $32 \%$ of participants with chronic diseases, more than $10 \%$ already developed CVD, $14.35 \%$ are smokers and more than $87 \%$ of the total population don't exercise on a regular basis.

Both diabetes and hypertension increase the risk of cardiovascular disease. According to WHO, the number of people with diabetes has risen from 108 million in 1980 to 422 million in 2014 worldwide. On the other hand, Research done by WHO revealed that the overall prevalence of raised blood pressure in adults aged 25 and over was around $40 \%$ in 2008 globally. Regarding our survey results, $40.37 \%$ of the populationare hypertensive, and $35.88 \%$ has diabetes. The prevalence of both diseases is increasing at an alarming rate ${ }^{[8]}$.

One of the cornerstones of prevention and management of CVD is patient self-awareness and proactive role in his own treatment. This is reflected by the population, and more specifically the at risk and diseased subjects, knowledge, and attitude towards the preventive practices of the disease.

In our survey, the healthy population knowledge regarding CVD risk factors is poor as illustrated in 
the results, for example only $36.93 \%$ knew that DM is a risk factor for CVD. On the contrary, subjects with risk factors have a sound knowledge about their disease and its relation with CVD, and the lowest knowledge was found in DM patients $65.44 \%$ knew that DM is a risk factor for CVD. These findings highlight the need for increasing our efforts to improve the general population awareness regarding CVD and preventive practices. Especially that physicians only contribute to around $30 \%$ of this knowledge, which is strikingly low.

Regarding the smokers and ex-smokers, there was a good percentage of them know that smoking is a risk factor for CVD, $79 \%$.

The channels we could reach the population with are the doctors, TV shows, awareness campaigns and through social media. And the main source that our population got theirpreventive medicine knowledge through is social media in $52.62 \%$ of total population. Health education sources based on our survey result were mainly from the social media (Facebook, Twitter, WhatsApp), while physicians came at last as a health educational source. This highlight the need to better utilize the social media to deliver the message of prevention as it is widely distributed and accepted plus the low cost and ease of reach, keeping in mind that the content should be monitored to prevent the delivery of wrong information. Also, we need to activate the role of the physician as a health advocate to improve the knowledge in pursuant to improve the attitude and ultimately the health of our society ${ }^{[7]}$.

This knowledge base was not translated to good attitude towards preventing risk factors through effective screening, routine follow-ups and checks, and good control of the chronic disease. For example, more than $72 \%$ of the healthy population don't measure their blood pressure routinely as recommended by the USPSTF and around 30\% of subjects with chronic disease do not follow up regularly. Physicians also contribute to this poor attitude by not offering to screen for risk factors.

One of the easiest and most affordable risk factors for adjustment is the lack of exercise. Compounding that with another risk factor will increase the risk for the development of CVD as the accumulation of more than one risk factor impose a higher risk for the development of CVD. Here we found that only small fraction of the healthy population is exercising and this percent is even lower among patients with chronic diseases. For example, those with high cholesterol level only $5.51 \%$ of them do exercise regularly

The most reported reason for the lack of follow up with physicians either for general checkups or disease monitoring is the lack of interest which deepens the need for improving the population awareness and their good health responsibility.

The cause of bad attitude and behavior in DM and general population rises from the lack of knowledge but for the others participant they have a good knowledge but yet they didn't translate it to proper behaviors and attitudes which tell us that we have to make more researches and studies regarding this matter.

\section{CONCLUSION}

We conduct this survey to determine and assess the knowledge, attitude and practice (KAP) of population toward the preventive medicine of CVD. In this study, we found the lack of knowledge in our healthy society and our diabetic participant that leads us to the fact that we should increase our effort in the clinic as doctors and improve the health education there, we should also increase our awareness campaigns, leaflet and Indicative Plates. So we can control the type, kind and amount of information that our population gets.

Regarding the remaining chronic patient participant, they have a good awareness but yet they didn't translate it to good attitude or good behaviors, and the same case appears with the smokers and exsmokers participant so its needs further investigations and researches.

To minimize the misleading health information that public are getting from social media we should increase the quantity of health knowledge materials (video, posters and tweets) released by authorized health channels. 


\section{REFRENCES}

1. American Board Of Preventive Medicine, last accessed at https://www.theabpm.org/aboutus.cfm on 27 December 2016

2. World Health Organization, Last accessed at http://www.euro.who.int/en/health-

topics/noncommunicable-

diseases/cardiovascular-

diseases/cardiovascular-diseases2/definitionof-cardiovascular-diseasesOn 27 Dec 2016

3. World Health Organization, Cardiovascular disease, Last accessed at http://www.who.int/mediacentre/factsheets/f s317/en/ on 27 December 2016

4. World Health Organization, About Cardiovascular Diseases, Last accessed http://www.who.int/cardiovascular_diseases/ about_cvd/en/ on 27 December 2016

5. Gaziano T, Reddy KS, Paccaud F, et al. Cardiovascular Disease. In: Jamison DT, Breman JG, Measham AR, et al., editors. Disease Control Priorities in Developing Countries. 2nd edition. Washington (DC): The International Bank for Reconstruction and Development / The World Bank; 2006. Chapter 33. Available from: https://www.ncbi.nlm.nih.gov/books/NBK11767/ Copublished by Oxford University Press, New York.https://www.ncbi.nlm.nih.gov/pubmed 126121190

6. Patel SA, Winkel M, Ali MK, Narayan KV, Mehta NK. Cardiovascular Mortality Associated With 5 Leading Risk Factors: National and State Preventable Fractions Estimated From Survey Data. Ann Intern Med. 2015;163:245-253. doi: 10.7326/M141753

7. World Health Organization, Global Health Observatory Data, Raised Blood Pressure, Last accessed at http://www.who.int/gho/ncd/risk_factors/blood_pressure_pre valence_text/en/ on 27 December 2016

8. World Health Organization, Last Accessed at http://www.who.int/mediacentre/factsheets/fs312/en/ on 27 December 2016 